\title{
Blinded by the Common Causes: Ischemic Monomelic Neuropathy; a Rare Complication after Vascular Access Creation
}

\author{
Awad Magbri*, Mariam El Magbri, Suhail Aftab, Karen Miller, Amanda Sinks, Stephen Ehret, Leisa Niki Canales and \\ Aimee Blankenship RN
}

Surgery and Vascular Center at Regency Park, USA

*Corresponding author: Awad Magbri, Surgery and Vascular Center at Regency Park, USA

\begin{abstract}
The authors discuss a case of a rare complications of vascular access creation in a hemodialysis patient "ischemic monomelic neuropathy". This is an ischemic axonal injury to the nerves supplying the left hand after arterio-venous graft placement for hemodialysis. The authors also discuss the diagnosis, pathophysiology and treatment of this rare vascular access complications that sometimes occurs in hemodialysis patients immediately following arterio-venous fistula or graft (AVF or AVG) placement.
\end{abstract}

Keywords: Ischemic Monomelic Neuropathy; Arterio-Venous Fistula or Graft; Hemodialysis; Nerve Conduction Studies; Steal Syndrome

\section{Case History}

The patient is 73-year Hispanic male with past medical history of hypothyroidism, type- 2 diabetes mellitus, hypertension, hyperlipidemia, and uremic encephalopathy diagnosed recently for which he was started on hemodialysis on May 2019. He had a left upper arm loop graft placed on June,13 2019, immediately after the surgery the patient complained of pain, numbness and tingling of the fingers and swelling of the of the left hand. He failed to followup with his surgeon. In the meantime, his symptoms got worse with increasing pain and swelling followed by loss of function of the left hand to the point that he could not grip objects with his hand.

He was referred to the Surgery and Vascular Center at Regency Park, Toledo, Ohio on the 8/28/2019 for fistulogram with a diagnosis of vascular steal syndrome. Clinical examination showed a thin elderly man in no acute distress. His vital signs were within normal limits. Examination of the cardio-vascular and respiratory systems were normal. He has a loop graft in the left upper arm with good thrill and bruits. His radial and ulnar pulses were palpable and within normal limits. The patient was not able to use the hand and fingers to grip objects or squeeze the examiner's hand. He has loss of sensation over the distributions of the medial and ulnar nerves with swelling of the left hand (Figures 1-5). The angiogram of the graft was normal (Figure 6). A preliminary diagnosis of ischemic monomelic neuropathy was entertained based on the clinical picture and the normal angiogram of the access. He was referred for nerve conduction studies and surgical consultation for possible ligature of the graft.

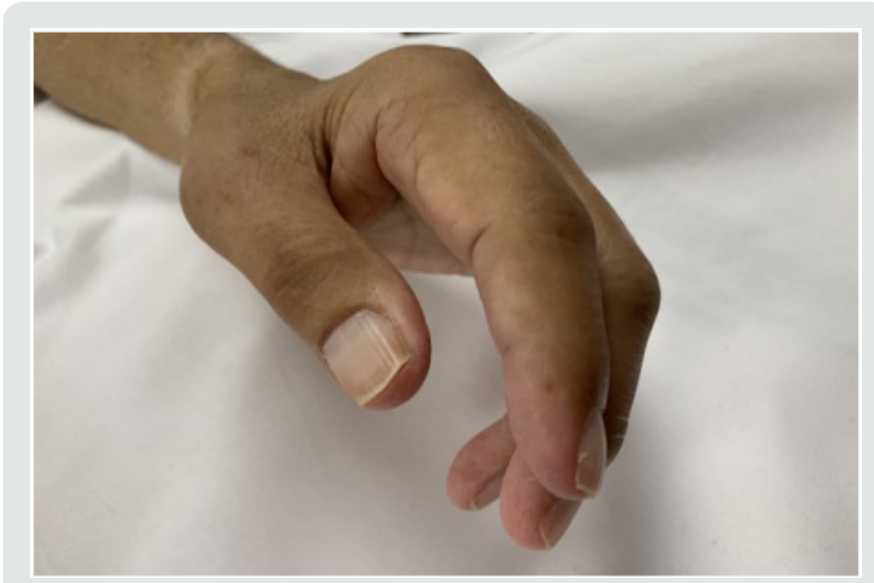

Figure 1. 


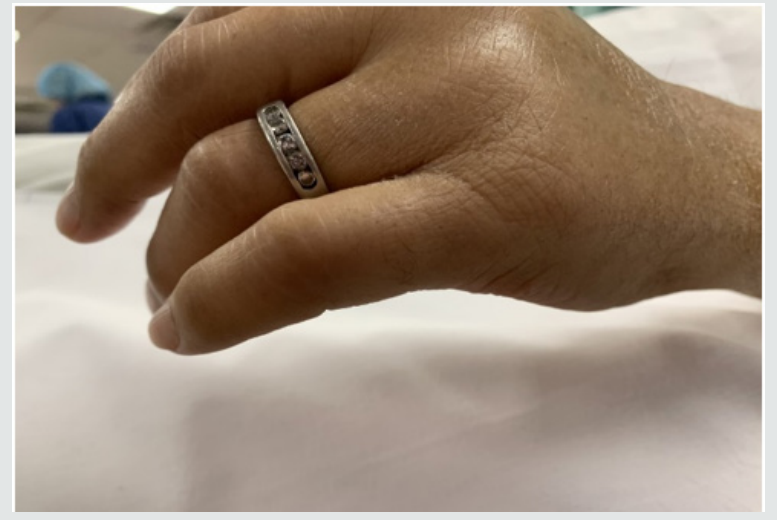

Figure 2.

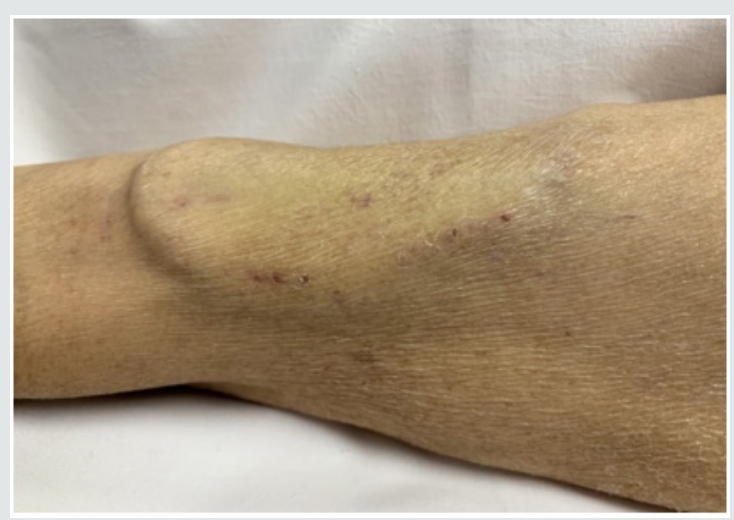

Figure 3.

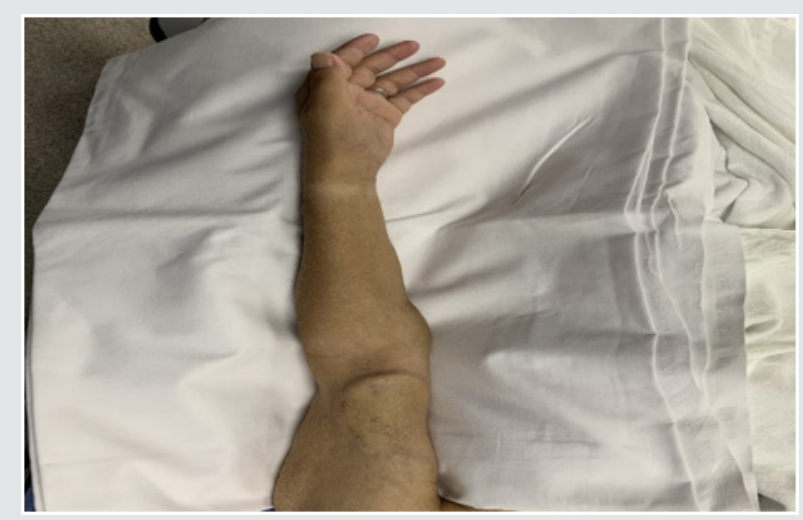

Figure 4.

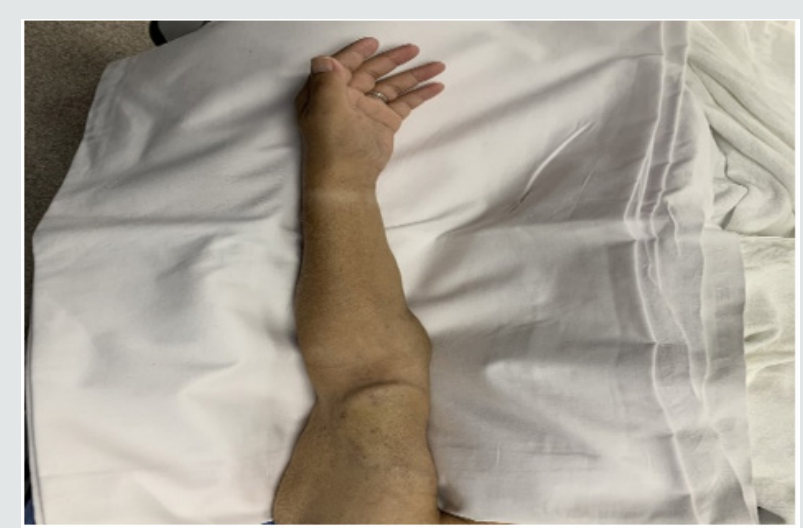

Figure 5.

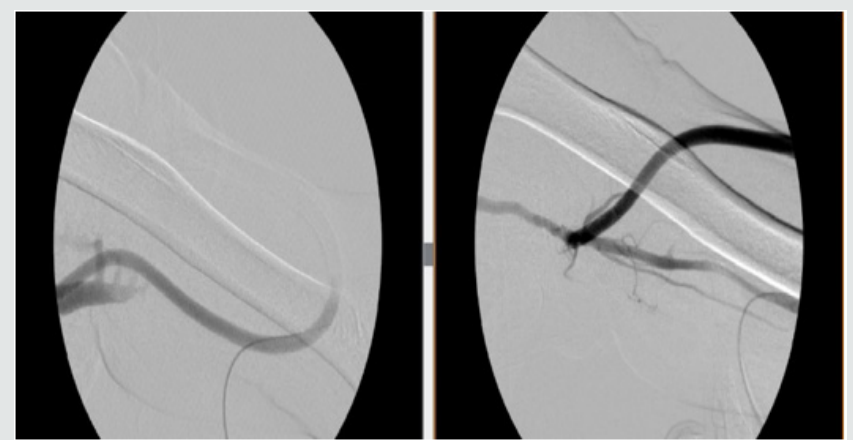

Figure 6.

\section{Case Discussion}

Ischemic monomelic neuropathy (IMN) is a combined sensory and motor impairment without major vascular or tissue necrosis. It is a form of ischemia of the nerves that supply the upper limbs especially the hand. It occurs immediately after vascular access creation for hemodialysis patients. It is a rare complication and caused by ischemic axonal loss of the nerves that supply the distal arms [1-3]. IMN is first reported by Wilbourn et al in the year 1983 [1]. IMN is under-recognized and mis-diagnosed, but its known incidence is 0.5 to $3 \%$ after vascular creations according to recent reports [3]. "What the mind cannot conceive the eyes cannot see". If a hemodialysis patient complains of hand pain after placement of arterio-venous fistula or graft, the physician needs to consider many diseases, including soft tissue swelling, wound hematoma, carpal tunnel syndrome, vascular steal syndrome, and IMN [4-6]. The most important factor in the diagnosis of IMN is to think of it and correlate and interpret the symptoms, signs of the clinical examination of the access and rule out vascular steal syndrome. Acute pain, weakness, and muscle paralysis immediately after AVF/AVG placement are common warning signs and symptoms for the presence of IMN. Since these symptoms are non-specific, after vascular access creation, the motor and sensory function of the operated hand should be examined carefully, and nerve conduction studies should be carried out urgently. Low amplitudes and reduced or even undetectable motor or sensory nerve conduction velocities in the presence of preservation of the vascular integrities of the hand are compatible with IMN. Axonal degeneration of the median, radial, and ulnar nerves can be observed [3].

Electromyogram (EMG) often show degeneration, including fibrillation potentials and motor unit loss. Past neuropathy, diabetes mellitus, atherosclerosis, upper arm vascular access, and female gender have increased risk for IMN [4,7-9]. These factors compromise the brachial artery which is the only blood supply to the distal arm. The most effective treatment of IMN is early recognition of the condition and immediate closure of the access; this increase the probability of recovery $[1,4]$. Early closure of the vascular access leads to partial or full recovery of the sensory and motor function of the hand [8]. The easiest way is to tie up the fistula or graft as soon as possible [5]. Better awareness and education of 
the surgeons and nephrologists should lead to early diagnosis and proper management of this dreadful complication (IMN) [4]. So, educating our minds to conceive the rare complications of vascular access surgeries are the best way to train the eyes to easily identify the complications before it is too late to help our patients.

\section{References}

1. Wilbourn AJ, Furlan AJ, Hulley W, Ruschhaupt W (1983) Ischemic monomelic neuropathy. Neurology 33(4): 447-451.

2. Thimmisetty RK, Pedavally S, Rossi NF, Fernandes JAM, Fixley J (2017) Ischemic monomelic neuropathy; diagnosis, pathogenesis, pathophysiology, and management. Kidney Int 2(1): 76-79.

3. Themann F, Kornhuber M (2011) Ishcemic monomelic neuropathy: a rare but important complication after hemodialysis access placement-a review. J Vasc Access 12(2): 113-119.
4. Kirksey L (2010) Ischemic monomelic neuropathy: an under-appreciated cause of pain and disability following vascular access surgery. J Vasc Access 11(2): 165-168.

5. Al Jaishi AA, Liu AR, Lok CE, Zhang JC, Moist LM (2017) Complications of the arteriovenous fistula: a systemic review. J AM Soc Nephrol 28(6): 1839-1850.

6. Feldman HI, Kobrin S, Wasserstein A (1996) Hemodialysis vascular access morbidity. J AM Soc Nephrol 7(4): 523-535.

7. Wodicka R, Isaacs J (2010) Ischemic monomelic neuropathy. J Hand Surg Am 35(5): 842-843.

8. Redfern AB, Zimmerman NB (1995) Neurologic and ischemic complications of upper extremity vascular access for dialysis. J Hand Surg AM 20(2): 199-204.

9. Miles AM (1999) Vascular steal syndrome and ischemic monomelic neuropathy; two variants of upper limb ischemia after hemodialysis vascular access surgery. Nephrol Dial Transplant 14(2): 297-230.

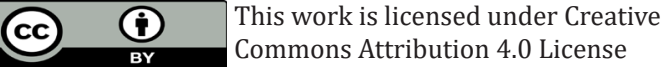

To Submit Your Article Click Here: Submit Article

DOI: 10.32474/ACR.2019.02.000141

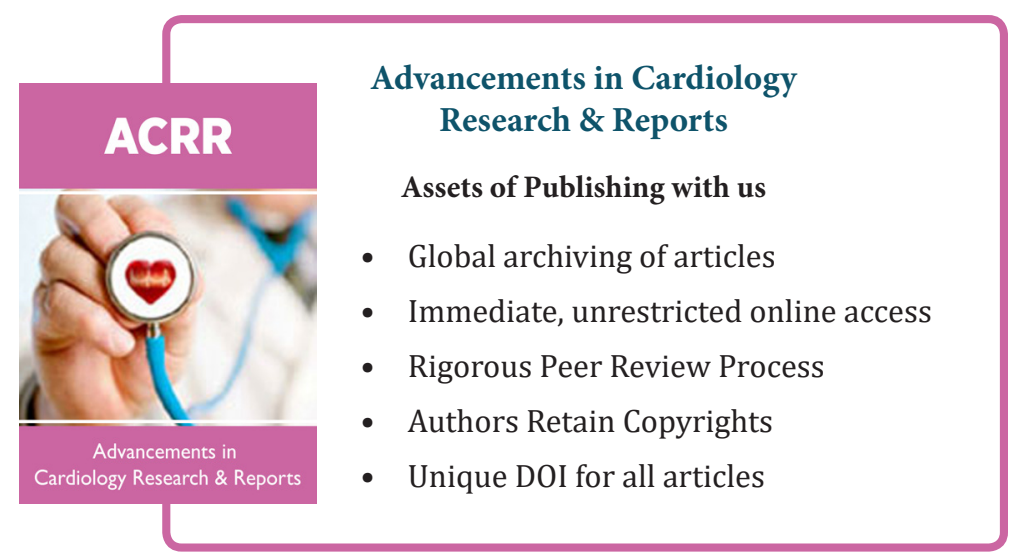

